Palavras chave:

Dendrometria Análise de regressão Floresta Amazônica

Histórico:

Recebido |4/| |/201 |

Aceito 29/04/2014

Keywords: Dendrometry

Regression analysis Amazon forest

Correspondência: forestengineer_ren@hotmail.com
Renato Bezerra da Silva Ribeiro', João Ricardo Vasconcellos Gama', Lia de Oliveira Melo'

\section{SECCIONAMENTO PARA CUBAGEM E ESCOLHA DE EQUAÇÕES DE VOLUME PARA A FLORESTA NACIONAL DO TAPAJÓS}

RESUMO: Objetivou-se, neste estudo, analisar diferentes comprimentos de seção para cubagem e ajustar modelos volumétricos para a estimativa da produção madeireira em uma área de manejo florestal na Floresta Nacional do Tapajós (FNT). Foram testados seis tratamentos de seccionamento em 152 toras de 12 espécies comerciais e os volumes obtidos comparados estatisticamente por meio da Análise de Variância (ANAVA), para a escolha do melhor método de seccionamento e o cálculo do volume real de 194 árvores amostra em diferentes classes de diâmetro. Foram ajustados 10 modelos matemáticos, para a floresta e para as espécies Manilkara huberi (Ducke) Chevalier (maçaranduba), Lecythis lurida (Miers) S.A.Mori (jarana) e Hymenaea courbaril L. (jatobá). Os critérios de escolha do melhor modelo foram: coeficiente de determinação ajustado em porcentagem $\left(\mathrm{R}_{\mathrm{aj}}^{2} \%\right)$, erro padrão da estimativa porcentual (Syx\%), significância dos parâmetros, normalidade dos resíduos, Fator de Inflação da Variância (VIF) e análise gráfica dos resíduos porcentuais. Não houve diferença estatística entre os métodos de seccionamento e, dessa forma, o comprimento total das toras foi o mais operacional em campo. Os modelos de Schumacher-Hall e Spurr, na forma logarítmica, foram que melhor estimaram o volume para as espécies em estudo e para área de manejo.

\section{SECTIONAL ANALYSIS FOR VOLUME DETERMINATION AND SELECTION OF VOLUME EQUATIONS FOR THE TAPAJOS NACIONAL FOREST}

ABSTRACT: The aim of this study was to analyze different sections lengths for volume determination, fitting of volumetric models for timber production estimation in an area of forest management in the Tapajós National Forest (FNT). Six treatments for sectioning were tested in 152 logs of 12 commercial species. The obtained volumes were statistically compared by analysis of variance (ANOVA) for the choice of the best method of sectioning and calculating the actual volume of 2,094 sample trees in different diameter commercial classes. Ten mathematical models were fitted to the whole data and to the species Manilkara huberi (Ducke) Chevalier (maçaranduba) Lecythis lurida (Miers) Samori (jarana) and Hymenaea courbaril L. (Jatobá). The criteria to choose the best model were adjusted coefficient of determination in percentage ( $\left.\mathrm{R}^{2} \mathrm{adj} \%\right)$, standard error of estimate in percentage (Syx\%), significance of the parameters, normality of residuals, Variance Inflation Factor (VIF) and residuals graphic distribution. There was no statistical difference between the methods of sectioning and thus the total length of the logs was more operational in the field. The models in logarithmic form of Schumacher and Hall and Spurr were the best to estimate the volume for the species and for the whole sample set. 


\section{INTRODUÇÃO}

Uma boa estimativa da volumetria de espécies comerciais é importante para a empresa florestal fazer uma correta avaliação do estoque de madeira e análise do potencial produtivo de suas florestas, como também é fundamental para o planejamento de colheita das empresas que executam ações de manejo florestal. Atualmente, com $\circ$ avanço nas discussões florestais, como o novo código florestal, as equações de volume de madeira tornaram-se uma ferramenta obrigatória para a estimativa do estoque de madeira dos planos de manejo.

Conforme a Resolução Conama $n^{\circ} 406$, de 02/02/2009 (BRASIL, 2009), todos os planos de manejo, a partir do segundo Plano Operacional Anual, deverão apresentar o volume comercial de árvores em pé, por meio de equações desenvolvidas especificamente para a floresta que está sendo manejada. E, dessa forma, melhorar a precisão do cálculo de volume, ou seja, estimar a volumetria mais real da floresta e, consequentemente, minimizar a possibilidade do comércio ilegal de créditos de volume de madeira, fato este que ainda existe na região oeste paraense.

A Floresta Nacional do Tapajós (FNT), criada pelo Decreto № 73.684, de 19 de fevereiro de 1974; está situada nos municípios de Belterra, Aveiro, Rurópolis e Placas, oeste do Estado do Pará. Por ser de uso sustentável, a FNT permite o manejo de parte de seus recursos naturais. Sendo assim, a Cooperativa Mista Flona Tapajós (COOMFLONA), constituída por comunitários residentes nesta unidade de conservação, vem desenvolvendo, desde 2005, ações de manejo florestal de produtos madeireiros e não madeireiros, sendo denominado de Projeto Ambé, em uma área de manejo florestal de 32.586,56 hectares (FERREIRANETO, 2008).

Nesse sentido, no presente trabalho, objetivouse a análise de seccionamento para cubem rigorosa e ajuste de modelos volumétricos para a estimativa da produção madeireira na área de manejo florestal do Projeto Ambé, Floresta Nacional do Tapajós.

\section{MATERIAL E MÉTODOS}

\section{Área de estudo}

O estudo foi realizado na área de manejo florestal pertencente ao Projeto Ambé no km 83 (BR- 163), localizado na Floresta Nacional do Tapajós, município de Belterra, Estado do Pará.
O clima da região é do tipo Ami (classificação de Köppen), com temperatura média anual de $25,5^{\circ} \mathrm{C}$, com umidade relativa média do ar de $90 \%$. A concentração de chuvas ocorre entre janeiro e maio, com uma precipitação média anual de $1.820 \mathrm{~mm}$. O relevo é pouco acidentado, com topografia variando de suavemente ondulada a ondulada. O solo é do tipo Latossolo Amarelo distrófico (INSTITUTO BRASILEIRO DO MEIO AMBIENTE E DOS RECURSOS NATURAIS RENOVÁVEIS - IBAMA, 2004). A Floresta Nacional do Tapajós situa-se na zona de Floresta Ombrófila Densa, tipo de vegetação dominante no norte do país (INSTITUTO BRASILEIRO DE GEOGRAFIA E ESTATÍSTICA - IBGE, 20I2), caracterizado pelo domínio de árvores de grande porte sob regime de temperaturas elevadas e precipitações distribuídas ao longo do ano (IBAMA, 2004).

\section{Coleta e análise dos dados}

$\mathrm{Na}$ execução do projeto de manejo florestal, uma das atividades de colheita é o romaneio de toras, com comprimento variando de $4,80 \mathrm{~m}$ a $7,50 \mathrm{~m}$, onde se mede a circunferência ou diâmetro nas extremidades da tora e, a partir desses dados, calcula-se o volume pelo método de Smalian ou geométrico como é denominado pelos órgãos ambientais (STERNADT, 200I).

Para verificar a possibilidade de utilização dos dados de romaneio no ajuste de modelos volumétricos, foram cubadas 152 toras de um total de 42 árvores, correspondente a 12 espécies comerciais para teste de seis tratamentos de seccionamento, sendo os comprimentos de secções iguais a: $\mathrm{TI}$ - comprimento total da tora (medida utilizada no romaneio); T2 - 2,0 m; T3 - 2,5 m; T4 - 3,0 m; T5 - 3,5 m e T6-4,0 m. Os volumes obtidos em cada tratamento foram calculados pela fórmula de Smalian. Os dados foram comparados por meio da Análise de Variância (ANAVA), utilizando o delineamento inteiramente casualizado.

Para o ajuste dos modelos volumétricos, foram selecionadas e cubadas as espécies comercializadas pela COOMFLONA, as quais totalizaram 194 árvores amostra, distribuídas em 30 espécies, em diferentes classes de DAP (diâmetro medido a I,30 m do solo) comercial $-50 \mathrm{~cm} \leq$ DAP $<170 \mathrm{~cm}$. Na Tabela I, representam-se as distribuições das árvores-amostra em classes de diâmetro - para a floresta e para as espécies: Maçaranduba (Manilkara huberi (Ducke) Chevalier), Jarana (Lecythis lurida (Miers) S.A.Mori) e Jatobá (Hymenaea courbaril L.), que é colhido a partir de $\mathrm{DAP} \geq 70 \mathrm{~cm}$. 
TABELA I Número de árvores-amostra por classes de diâmetro para a floresta e para as espécies maçaranduba, jarana e jatobá na área de manejo florestal do Projeto Ambé, Flona Tapajós.

TABLE I Number of sample trees by diameter classes for the forest and for maçaranduba, jarana and jatoba species in the forest management area of Ambe Project, Tapajós National Forest.

\begin{tabular}{lccccccc}
\hline \multirow{2}{*}{ Amostra } & \multicolumn{7}{c}{ Centro de classe de diâmetro } \\
\cline { 2 - 7 } & 60 & 80 & 100 & 120 & 140 & 160 & Total \\
\hline Floresta & 33 & 38 & 38 & 37 & 38 & 10 & 194 \\
Maçaranduba & 9 & 12 & 12 & 12 & 3 & 48 \\
Jarana & 12 & 13 & 14 & 12 & 1 & 52 \\
Jatobá & 19 & 20 & 20 & 13 & 1 & 73 \\
\hline
\end{tabular}

\section{Ajuste das equações de volume}

Após o cálculo dos volumes reais das toras com casca pela fórmula de Smalian, foram ajustados 10 modelos matemáticos, para a floresta e para as espécies em estudo (Tabela 2).

Como critérios para a escolha dos melhores modelos, foram avaliados: coeficientes de determinação ajustados em porcentagem $\left(R_{a j}^{2} \%\right)$, erro padrão da estimativa em porcentual (Syx\%) e análise gráfica dos resíduos porcentuais. Contudo, foi analisada também a significância dos parâmetros pelo Teste t a $5 \%$ de probabilidade, normalidade dos resíduos por meio do Teste Kolmogov-Smirnov a 5\% de probabilidade e Fator de Inflação da Variância (para os modelos com mais de uma variável independente) para verificar problemas de multicolinearidade.

Para comparação das equações logarítmicas com as não logarítmicas, o erro padrão de estimativa e o coeficiente de determinação foram recalculados, conforme Scolforo (1993). Os testes aplicados e os modelos volumétricos (ajustados pelo método dos mínimos quadrados ordinários - MMQO) foram calculados por meio dos Softwares Excel 2010 e Action 2.5.

\section{Validação das equações}

Foi separado um banco de dados à parte, com 120 árvores de 15 espécies para a floresta, 40 árvores de maçaranduba, 40 árvores de jarana e 30 árvores de Jatobá, as quais não fizeram parte dos ajustes dos modelos e aplicado o Teste Qui-Quadrado a 5\% de probabilidade para comparar o volume real com o volume estimado pelas melhores equações selecionadas.

\section{RESULTADOS E DISCUSSÃO}

\section{Cubagem de toras}

Cada árvore apresentou em média quatro toras e os volumes médios e desvios para cada tratamento aplicado foram: 8,86 $\mathrm{m}^{3} \pm 3,54 \mathrm{~m}^{3}$ (TI); 8,60 $\mathrm{m}^{3} \pm 3,6 \mathrm{I}$ $\mathrm{m}^{3}$ (T2); 8,62 $\mathrm{m}^{3} \pm 3,61 \mathrm{~m}^{3}$ (T3); 8,66 $\mathrm{m}^{3} \pm 3,63 \mathrm{~m}^{3}$ (T4); 8,67 $\mathrm{m}^{3} \pm 3,63 \mathrm{~m}^{3}$ (T5); e 8,68 $\mathrm{m}^{3} \pm 3,63 \mathrm{~m}^{3}$ (T6). De acordo com a análise de variância, os volumes obtidos não diferiram estatisticamente (Tabela 3).

Todavia, o tratamento I foi escolhido considerando a possibilidade dos manejadores utilizarem os dados de romaneio no ajuste dos modelos para estimar o volume de árvores de unidades de produção anual contíguas e, assim, ter um banco de dados mais robusto sem a necessidade de uma coleta específica, que alteraria a rotina operacional da equipe de romaneio.

TABELA 2 Modelos volumétricos ajustados para a área de manejo florestal do Projeto Ambé, Floresta Nacional do Tapajós.

TABLE 2 Volumetric models fitted for the management area of Ambé Project, Tapajos National Forest.

\begin{tabular}{clc}
\hline Número & \multicolumn{1}{c}{ Modelos } & Autor \\
\hline I & $\ln (\mathrm{V})=\beta_{0}+\beta_{1} \cdot \ln (\mathrm{D})+\beta_{2} \cdot \ln (\mathrm{H})+\varepsilon$ & Schumacher-Hall logarítmizado \\
2 & $\ln (\mathrm{V})=\beta_{0}+\beta_{1} \cdot \ln (\mathrm{D})+\beta_{2} \cdot \ln \left(\mathrm{D}^{2}\right)+\beta_{3} \cdot \ln (\mathrm{H})+\beta_{4} \cdot \ln \left(\mathrm{H}^{2}\right)+\varepsilon$ & Prodan \\
3 & $\mathrm{~V}=\beta_{0}+\beta_{1} \cdot \mathrm{D}+\beta_{2} \cdot \mathrm{D}^{2}+\beta_{3} \cdot \mathrm{D} \cdot \mathrm{H}+\beta_{4} \cdot \mathrm{D}^{2} \cdot \mathrm{H}+\beta_{5} \cdot \mathrm{H}+\varepsilon$ & Stoate \\
4 & $\mathrm{~V}=\beta_{0}+\beta_{1} \cdot \mathrm{D}^{2}+\beta_{2} \cdot \mathrm{D}^{2} \cdot \mathrm{H}+\beta_{3} \cdot \mathrm{H}+\varepsilon$ & Spurr (variável combinada) \\
5 & $\mathrm{~V}=\beta_{0}+\beta_{1} \cdot \mathrm{D}^{2} \cdot \mathrm{H}+\varepsilon$ & Spurr logaritmizado \\
6 & $\ln (\mathrm{V})=\beta_{0}+\beta_{1} \cdot \ln \left(\mathrm{D}^{2} \mathrm{H}\right)+\varepsilon$ & Näslund \\
7 & $\mathrm{~V}=\beta_{1} \cdot \mathrm{D}^{2}+\beta_{2} \cdot \mathrm{D}^{2} \cdot \mathrm{H}+\beta_{3} \cdot \mathrm{D} \cdot \mathrm{H}^{2}+\beta_{4} \cdot \mathrm{H}^{2}+\varepsilon$ & Meyer modificado \\
8 & $\mathrm{~V}=\beta_{0}+\beta_{1} \cdot \mathrm{D}+\beta_{2} \cdot \mathrm{D}^{2}+\beta_{3} \cdot \mathrm{D} \cdot \mathrm{H}+\beta_{4} \cdot \mathrm{D}^{2} \cdot \mathrm{H}+\varepsilon$ & Compreensiva \\
9 & $\mathrm{~V}=\beta_{0}+\beta_{1} \cdot \mathrm{D}+\beta_{2} \cdot \mathrm{D} \cdot \mathrm{H}+\beta_{3} \cdot \mathrm{D}^{2}+\beta_{4} \cdot \mathrm{H}+\beta_{5} \cdot \mathrm{D}^{2} \cdot \mathrm{H}+\varepsilon$ & Dissescu-Meyer \\
\hline
\end{tabular}

$\mathrm{V}=$ volume comercial com casca, em m $\mathrm{m}^{3} \mathrm{D}=$ diâmetro à altura de $\mathrm{I}, 30 \mathrm{~m}$ (DAP), em cm; $\mathrm{H}=$ altura comercial, em m; $\beta_{0} \beta_{1} \beta_{2} \beta_{3} \beta_{4} \beta_{5}=$ parâmetros de regressão; $\ln ()=$ logaritmo neperiano; e $\varepsilon=$ erro aleatório. 
TABELA 3 Resultado da análise de variância aplicado aos tratamentos de seccionamento de toras, área de manejo florestal do Projeto Ambé, Floresta Nacional do Tapajós.

TABLE 3 Result of analysis of variance applied to treatments sectioning logs, management area of Ambé Project, Tapajos National Forest.

\begin{tabular}{lccccc}
\hline $\begin{array}{l}\text { Fonte da } \\
\text { variação }\end{array}$ & GL & SQ & QM & $F_{\text {calc }}$ & $F_{\text {tab }(5, \infty)}$ \\
\hline Tratamentos & 5 & 0,4821934 & 0,096439 & $0,1263^{\text {ns }}$ & 2,21 \\
Resíduos & 906 & 691,58062 & 0,763334 & & \\
Total & 911 & 692,06282 & & & \\
\hline
\end{tabular}

ns = não significativo a um nível de $95 \%$ de probabilidade.

\section{Equações de volume para a floresta}

Dentre os 10 modelos testados, os dois que melhor ajustaram aos dados observados de volume para a floresta foram: Schumacher-Hall (SH) e Spurr (SP), ambos na forma logarítmica (Tabela 4). Os coeficientes de determinação ajustado $\left(\mathrm{R}_{\text {aj }}^{2} \%\right.$ ) variaram de $93,48 \%$ a $94,10 \%$ e o erro padrão da estimativa de $17,06 \%$ a $17,12 \%$. $\mathrm{O}$ modelo de $\mathrm{SH}$ foi o único que não teve problema de multicolinearidade (VIF < I0), e somente SP na forma aritmética e logarítmica que não houve este tipo de análise, por apresentar apenas uma variável independente.

Em estudo com espécies de valor comercial em Paragominas, Estado do Pará, Silva et al. (20I I) também presenciaram ausência de multicolinearidade nas variáveis independentes de Schumacher-Hall e elegeram o mesmo como a melhor equação para a estimativa volumétrica naquela ocasião. Monteiro (2009) e Rolim et al. (2006) também tiveram o modelo de Schumacher e Hall como o melhor na estimativa volumétrica de seus estudos. Rolim et al. (2006) trabalharam na Floresta Nacional de Tapirapé-Aquirí, localizada na Serra dos Carajás (PA) e Monteiro (2009) em uma floresta primária no sudeste paraense, especificamente no município de Paragominas, Pará.

Os parâmetros das equações de $\mathrm{SH}$ e SP também foram significativos pelo Teste $\mathrm{t}$ a $5 \%$ de probabilidade (Tabela 4) e a normalidade dos resíduos comprovadas pelo Teste de Kolmogorov-Smirnov a 5\% de probabilidade, sendo $\mathrm{SH}(p=0,4676)$ e SP $(p=0,662 \mathrm{I})$.

Em relação aos gráficos dos resíduos, verificaram-se pontos bem distribuídos ao longo dos diâmetros nos dois melhores modelos, o que é desejado para as equações volumétricas (Figura I).
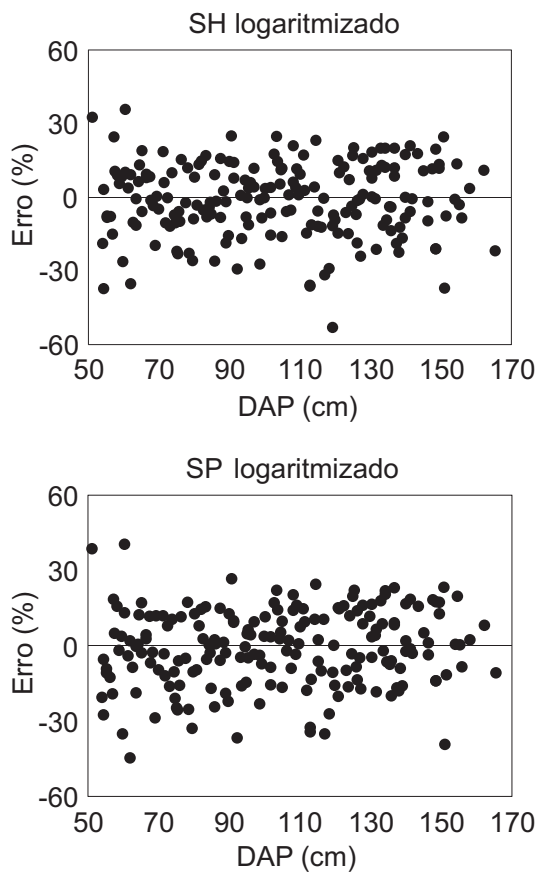

FIGURA I Distribuição gráfica dos resíduos para a floresta com os dois melhores modelos ajustados.

FIGURE I Graphic distribution of the residuals of the forest with the two best models fitted for the management area.

Para Silva et al. (1984), em estudo sobre equações de volume no km 67 na FNT, os modelos que melhor ajustaram os dados para a floresta foram: Schumacher e Hall logaritmizado como de dupla entrada e Husch como de simples entrada. Posteriormente, Moura (1994), também na FNT, concluiu em seu trabalho que os melhores modelos para floresta foram: Schumacher e Hall e Prodan, ambos na forma não-linear.

TABELA 4 Resultado dos ajustes com os dois melhores modelos para a floresta.

TABLE 4 Result of the two best models fitted for the forest.

\begin{tabular}{|c|c|c|c|c|c|c|c|c|}
\hline \multirow{2}{*}{ Modelo } & \multicolumn{6}{|c|}{ Coeficientes / Significância dos parâmetros } & \multirow{2}{*}{$\mathrm{R}_{\mathrm{aj}}^{2} \%$} & \multirow{2}{*}{ Sy.x\% } \\
\hline & $\beta_{0}$ & p-valor & $\beta_{1}$ & p-valor & $\beta_{2}$ & $\mathrm{p}$-valor & & \\
\hline SH logaritmizado & $-7,9655227$ & $2,4083 \times 10^{-94}$ & I,7928I5। & $1,9335 \times 10^{-108}$ & 0,5934104 & $9,2503 \times 10^{-21}$ & 94,12 & 17,06 \\
\hline SP logaritmizado & $-8,2863833$ & $1,8744 \times 10^{-98}$ & 0,8461009 & $4,7769 \times 10^{-116}$ & - & - & 93,49 & 17,12 \\
\hline
\end{tabular}

$\mathrm{R}_{\text {aj }}^{2} \%$ = coeficiente de determinação ajustado em percentagem; Syx\% = erro-padrão da estimativa em percentagem; $p$-valor = probabilidade pelo teste t a $5 \% ; \beta_{0}, \beta_{1}, \beta_{2}=$ parâmetros da regressão. 
No presente estudo, os modelos logaritmizados seguiram todos os pressupostos desejados para a análise de regressão e, consequentemente, mais eficientes que os modelos aritméticos. Silva et al. (20I I) explicam que a distribuição amostral dos dados de volume de madeira tende a apresentar curva assimétrica para a direita e a transformação logarítmica resolve esse problema, aumentando a eficiência dos parâmetros estimados pelo método de mínimos quadrados ordinários.

O modelo de Schumacher-Hall, na forma logarítmica, tem sido o mais difundido, em razão de suas propriedades estatísticas, uma vez que resulta quase sempre em estimativas não-tendenciosas e $\circ$ modelo de Spurr atribuída mais pela facilidade de ajustamento (CAMPOS; LEITE, 2002).

\section{Equações de volume para a Maçaranduba}

Para a espécie maçaranduba, os modelos de Schumacher-Hall e Spurr também foram os que apresentaram as melhores estimativas de volume. $\mathrm{O}$ coeficiente de determinação ajustado $\left(\mathrm{R}_{\mathrm{aj}}^{2} \%\right)$ para $\mathrm{SH}$ foi de $93,54 \%$ e para SP $90,70 \%$, enquanto que o erro padrão da estimativa (Syx\%) variou entre $13,64 \%$ e 16,29\% (Tabela 5).

O Teste de Kolmogorov-Smirnov indicou normalidade para os resíduos dos dois modelos, tendo as probabilidades $p=0,9407$ para $\mathrm{SH}$ e $p=0,5004$ para SP. O Fator de inflação da Variância para as variáveis independentes de $\mathrm{SH}$ foi I,009 para ambas, indicando não haver problema de multicolinearidade. Além disso, todos os parâmetros foram significativos pelo Test $\mathrm{t}$ (Tabela 5).

No estudo de Monteiro (2009), o melhor modelo para a espécie maçaranduba foi o de Schumacher e Hall, com um coeficiente de determinação de $88 \%$ e uma amostra de 210 indivíduos, divididos em sete classes diamétricas. Para Silva et al. (1984), os modelos que apresentaram os melhores ajustes para a maçaranduba, foram Schumacher-Hall (dupla entrada) e Huch (simples entrada). Enquanto que, para Moura (1994), o melhor modelo foi o de Meyer.

A distribuição gráfica dos resíduos se comportou de forma semelhante entre os modelos SH e SP com erros percentuais abaixo de $40 \%$, sendo que para SP os pontos foram mais dispersos do que de SH (Figura 2). E, dessa forma, $\mathrm{SH}$ foi melhor para a estimativa volumétrica para a maçaranduba.
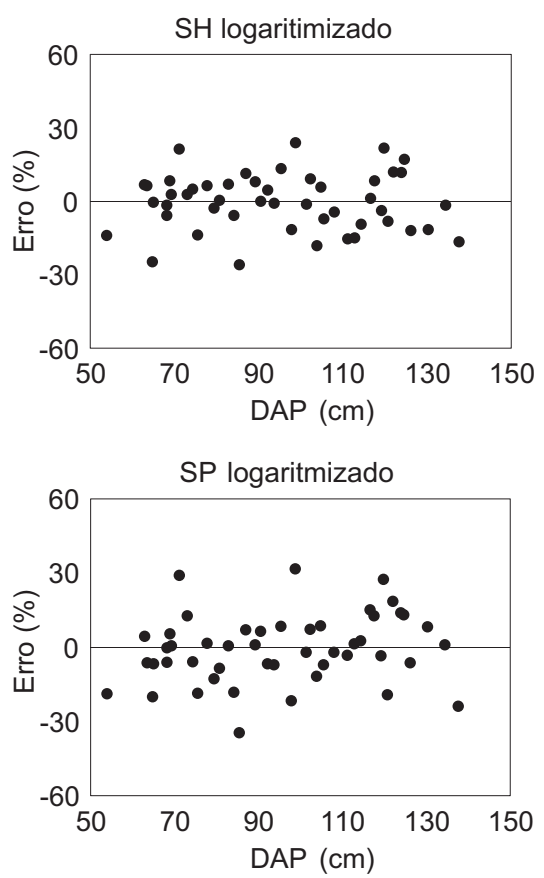

FIGURA 2 Distribuição gráfica dos resíduos para a espécie maçaranduba com os dois melhores modelos ajustados para a área de manejo florestal.

FIGURE 2 Graphic distribution of the residuals of the maçaranduba species with the two best models fitted for the management area.

\section{Equações de volume para a Jarana}

Para a jarana, os coeficientes de determinação ajustados para os modelos de Schumacher-Hall e Spurr, foram $94,39 \%$ e $93,94 \%$, respectivamente, e os erros padrão da estimativa, de $12,74 \%$ a $13,84 \%$ (Tabela 6). Os resíduos das melhores equações apresentaram normalidade pelo Teste de Kolmogorov-Smirnov, com $p=0,562$ I para SH e $p=0,7652$ para SP.

A multicolinearidade não apresentou problemas para Schumacher-Hall com VIF $=I, 058$ para ambas variáveis independentes. E para todos os parâmetros dos dois melhores modelos houve significância pelo Teste $\mathbf{t}$ (Tabela 6).

TABELA 5 Resultado dos ajustes com os dois melhores modelos para a maçaranduba.

TABLE 5 Result of the two best models fitted for the maçaranduba species.

\begin{tabular}{|c|c|c|c|c|c|c|c|c|}
\hline \multirow{2}{*}{ Modelo } & \multicolumn{6}{|c|}{ Coeficientes / Significância dos parâmetros } & \multirow{2}{*}{$\mathrm{R}_{\mathrm{aj}}^{2} \%$} & \multirow{2}{*}{ Sy.x\% } \\
\hline & $\beta_{0}$ & $\mathrm{p}$-valor & $\beta_{1}$ & $\mathrm{p}$-valor & $\beta_{2}$ & $\mathrm{p}$-valor & & \\
\hline SH logaritmizado & $-7,6088 \mid 15$ & $2,1577 \times 10^{-23}$ & 1,7911272 & $5,8016 \times 10^{-28}$ & 0,4596757 & $1,9463 \times 10^{-06}$ & 93,54 & 13,64 \\
\hline SP logaritmizado & $-7,9984617$ & $9,0122 \times 10^{-22}$ & $0,8|8522|$ & $1,3960 \times 10^{-25}$ & - & - & 90,70 & 16,29 \\
\hline
\end{tabular}


A distribuição gráfica dos resíduos entre os melhores modelos apresentaram resultados com tendência semelhantes, com erros percentuais abaixo de $30 \%$ e com alguns pontos de superestimativa e distantes de zero nos maiores diâmetros (Figura 3). Entretanto, devido a todas as outras análises, os modelos de SH e SP foram os mais confiáveis para estimar o volume dessa espécie.
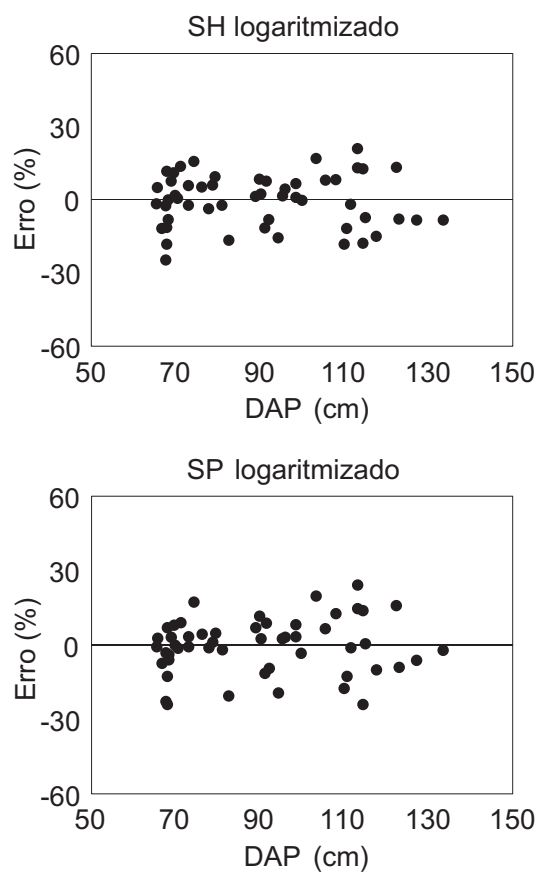

FIGURA 3 Distribuição gráfica dos resíduos para a espécie jarana com os dois melhores modelos ajustados.

FIGURE 3 Graphic distribution of the residuals of the jarana species with the two best fitted models.

Silva et al. (1984) definiram também em seus estudos que o modelo mais adequado para estimativa do volume da jarana foi de Schumacher-Hall, assim como Moura (1994), o qual não descartou a possibilidade de uso dos modelos de Prodan e Meyer.

\section{Equações de volume para o Jatobá}

$\mathrm{Na}$ Tabela 7, estão listados os resultados dos coeficientes de determinação dos melhores modelos para estimativa do volume da espécie jatobá. Verificase que os valores ficaram acima de $90 \%$, tendo o modelo de Schumacher-Hall, o melhor resultado $\left(R^{2} a j=93,43 \%\right)$, seguido pelo modelo de Spurr $\left(R^{2} a j=\right.$ 9।,44\%). Em relação ao erro padrão da estimativa percentual, os valores ficaram entre $10,13 \%$ e I I,28\%.

O VIF para o modelo de $\mathrm{SH}$ foi de I,008 para ambas as variáveis independentes. Houve normalidade dos resíduos pelo Teste de Kolmorogorov-Smirnov para os dois modelos, com probabilidades $p=0,276 \mathrm{I}$ para $\mathrm{SH}$ e $p=0,7928$ para SP. E todos os parâmetros tiveram significância pelo Teste $t$ (Tabela 7).

A espécie jatobá também foi estudada por Monteiro (2009), para a determinação de uma equação de volume específica, em uma área localizada no município de Paragominas e os resultados apontaram que o melhor modelo de dupla entrada para a estimativa dos volumes foi a de Spurr na forma logarítmica, com coeficiente de determinação de $90 \%$.

Por meio da Figura 4, pode-se observar a representação gráfica residual dos modelos. A análise mostra que os modelos apresentaram erros abaixo de $30 \%$, com tendência a superestimar os volumes nos maiores diâmetros. Contudo, optou-se pelo modelo de $\mathrm{SH}$, por ter pontos menos dispersos.

TABELA 6 Resultado dos ajustes com os dois melhores modelos para a jarana, na área de manejo florestal.

TABLE 6 Result of the two best models fitted for the jarana species in the management area.

\begin{tabular}{ccccccccc}
\hline \multirow{2}{*}{ Modelo } & \multicolumn{9}{c}{ Coeficientes / Significância dos parâmetros } & \multirow{2}{*}{$\mathrm{R}_{\mathrm{aj}}^{2} \%$} & \multirow{2}{*}{ Sy.x\% } \\
\cline { 2 - 7 } & $\beta_{0}$ & $\mathrm{p}$-valor & $\beta_{1}$ & $\mathrm{p}$-valor & $\beta_{2}$ & $\mathrm{p}$-valor & \\
\hline SH logaritmizado & $-7,9423306$ & $\mathrm{I}, 4525 \times 10^{-28}$ & $\mathrm{I}, 7303838$ & $6,9092 \times 10^{-29}$ & 0,6823796 & $3,9555 \times 10^{-14}$ & 94,39 & 12,74 \\
SP logaritmizado & $-7,8835633$ & $4,7381 \times 10^{-28}$ & 0,8146045 & $2,5861 \times 10^{-32}$ & - & - & 93,94 & 13,84 \\
\hline
\end{tabular}

TABELA 7 Resultado dos ajustes com os dois melhores modelos para a espécie jatobá.

TABLE 7 Result of the two best models fitted for the jatobá species.

\begin{tabular}{|c|c|c|c|c|c|c|c|c|}
\hline \multirow{2}{*}{ Modelo } & \multicolumn{6}{|c|}{ Coeficientes / Significância dos parâmetros } & \multirow{2}{*}{$\mathrm{R}_{\mathrm{aj}}^{2} \%$} & \multirow{2}{*}{ Sy.x\% } \\
\hline & $\beta_{0}$ & $\mathrm{p}$-valor & $\beta_{1}$ & p-valor & $\beta_{2}$ & $\mathrm{p}$-valor & & \\
\hline SH logaritmizado & $-7,2142005$ & $1,2881 \times 10^{-29}$ & I,7797| 46 & $1,19308 \times 10^{-42}$ & 0,4486902 & $1,7043 \times 10^{-06}$ & 93,43 & 10,13 \\
\hline SP logaritmizado & $-8,020034$ & $3,9533 \times 10^{-32}$ & 0,8367190 & $7,5116 \times 10^{-40}$ & - & - & 91,44 & II,28 \\
\hline
\end{tabular}



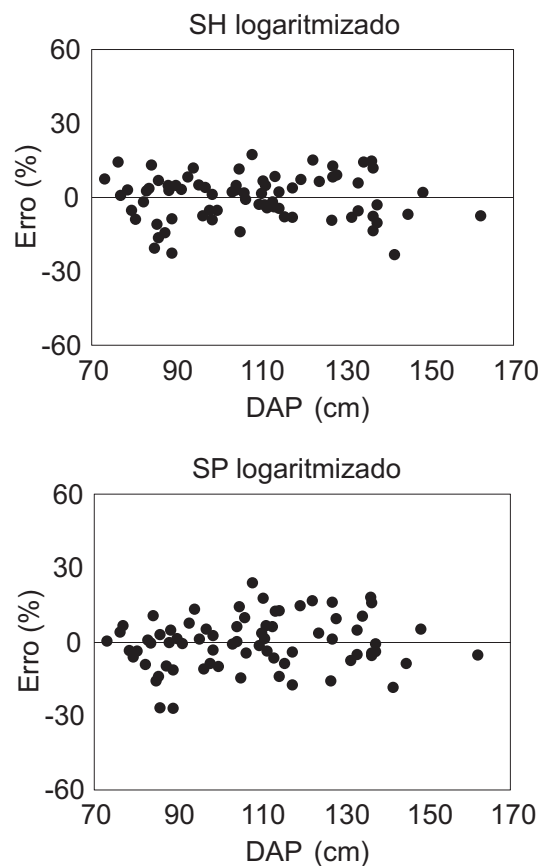

FIGURA 4 Distribuição gráfica dos resíduos para a espécie jatobá com os dois melhores modelos ajustados.

FIGURE 4 Graphic distribution of the residuals of the jatobá species with the two best fitted models.

Para Silva et al. (1984), os modelos de Schumacher-Hall e Husch foram os mais adequados à estimativa do volume do jatobá. Já, Moura (1994), optou pelo modelo de Prodan como o mais apropriado para a espécie.

\section{Validação das equações}

O teste Qui-quadrado $\left(\chi^{2}\right)$ aplicado para comparar os volumes observados com $\circ$ volumes estimados pelas melhores equações volumétricas, tanto para floresta, quanto para as espécies maçaranduba, jarana e jatobá, mostrou não haver diferença significativa a um nível de $5 \%$ de probabilidade (Tabela 8 ).

TABELA 8 Resultado do Teste Qui-quadrado a 5\% de probabilidade para os volumes observados e estimados pelas melhores equações na área de manejo do Projeto Ambé.

TABLE 8 Result of the Chi-square test at 5\% probability for the observed and estimated volumes by the best equations in the management area of Ambe Project.

\begin{tabular}{ccccc}
\hline & Floresta & Maçaranduba & Jarana & Jatobá \\
\hline Qcal & 1,8300 & 1,3745 & 5,4913 & 1,9378 \\
(p)* & 0,3916 & 0,2885 & 0,8608 & 0,6205 \\
\hline
\end{tabular}

$*(p) \geq 0,05$ não houve diferença significativa entre os volumes.
Dessa forma, aceita-se a hipótese nula de que o modelo de Schumacher-Hall é válido para as estimativas volumétricas das espécies em estudo e para a floresta.

\section{CONCLUSÃO}

É adequado o uso do comprimento de tora para o cálculo de volume real, podendo-se utilizar dados de romaneio para ajuste de modelos volumétricos.

Os dois modelos volumétricos que mais se destacaram na estimativa do volume da floresta e das espécies (maçaranduba, jarana e jatobá), foram: Schumacher-Hall e Spurr, ambos na forma logarítmica.

\section{AGRADECIMENTOS}

Agradecemos à Cooperativa Mista da FLONA Tapajós e, em especial, aos manejadores pelo apoio recebido durante a coleta de dados.

\section{REFERÊNCIAS}

BRASIL. Ministério do Meio Ambiente. Resolução Conama $n^{\circ}$ 406, de 02 de fevereiro de 2009. Estabelece parâmetros técnicos a serem adotados na elaboração, apresentação, avaliação técnica e execução de Plano de Manejo Florestal Sustentável-PMFS com fins madeireiros, para florestas nativas e suas formas de sucessão no bioma Amazônia. Brasília, 2009. Disponível em: <http://www.mma.gov.br/ port/conama/legiabre.cfm?codlegi $=597>$. Acesso em: 10 dez. 2013.

CAMPOS, J. C. C.; LEITE, H. G. Mensuração florestal: perguntas e respostas. Viçosa, MG: UFV, 2002. 407 p.

FERREIRA-NETO, P. S. Projeto Ambé: manejando a floresta e colhendo conhecimentos. Brasília: Ministério do Meio Ambiente, 2008. 85 p.

INSTITUTO BRASILEIRO DE GEOGRAFIA E ESTATÍSTICA. Manual técnico da vegetação brasileira. 2. ed. Rio de Janeiro, 20I2. 27I p.

INSTITUTO BRASILEIRO DO MEIO AMBIENTE E DOS RECURSOS NATURAIS RENOVÁVEIS. Floresta nacional do Tapajós: plano de manejo. Brasília, 2004. 373 p.

MONTEIRO, L. A. S. Equações de volume geral e para dez espécies dominantes em uma floresta primária no sudeste do Pará. 2009. 66 p. Dissertação (Mestrado em Ciências Florestais) - Universidade Federal Rural da Amazônia, Belém, 2009. 
MOURA, J. B. Estudo da forma do fuste e comparação de métodos de estimativa volumétrica de espécies florestais da Amazônia brasileira. 1994. II4 p. Dissertação (Mestrado em Engenharia Florestal) Universidade Federal do Paraná, Curitiba, 1994.

ROLIM, S. G.; COUTO, H. T. Z.; JESUS, R. M.; FRANÇA, J. T. Modelos volumétricos para a floresta nacional do TapirapéAquirí, Serra dos Carajás, PA. Acta Amazonica, Manaus, v. 36, n. I, p. 107-II4, 2006.

SCOLFORO, J. R. S. Mensuração florestal 3: relações quantitativas em volume, peso e a relação hipsométrica. Lavras: ESAL/FAEPE, 1993. 292 p.
SILVA, E. N.; SANTANA, A. C.; QUEIROZ, W. T.; SOUSA, R. J. Estimação de equações volumétricas para árvores de valor comercial em Paragominas, Estado do Pará. Amazônia: Ciência \& Desenvimento, Belém, v. 7, n. 13, p. 7-18, jul./ dez. $201 \mathrm{I}$.

SIlVA, J. N. M.; CARVAlHO, J. O. P.; LOPES, J. C. A.; CARVALHO, M. S. P. Equações de volume para a floresta nacional do Tapajós. Boletim de Pesquisa Florestal, Curitiba, n. 8/9, p. 50-63, 1984.

STERNADT, G. H. Cubagem de toras de mogno: comparação do processo do IBAMA e o adotado por madeireiras. Brasília: IBAMA, 200I. I00 p. 